\title{
Deflection microwave amplifier with field-emitter arrays
}

Cha-Mei Tang

Plasma Physics Division, Naval Research Laboratory, Washington, DC 20375-5346

Y. Y. Lau

Department of Nuclear Engineering, University of Michigan, Ann Arbor, Michigan 48109-2104

and Omega P, Inc., 2008 Yale Station, New Haven, Connecticut 06520

T. A. Swyden

FM Technologies, Inc., Fairfax, Virginia 22032

A new class of microwave and millimeter-wave amplifiers, based on the deflection of collimated microscopic electron beams with high-current density and low voltage from field-emitter arrays, was analyzed. The deflection concept may be applied in two ways; as microelectronic amplifiers or as bunched beam cathodes to power conventional amplifier configurations such as klystrodes and traveling wave tubes. We found that the frequency $f_{T}$, at which the short-circuit current gain attains unit magnitude, depends only on the electron beam energy, current density, and emittance, and is independent of beamwidth and total current. The results indicate that field-emitter arrays have the potential of making wideband deflection amplifiers at frequencies up to tens of $\mathrm{GHz}$. (C) 1994 American Institute of Physics.

There is sustained interest in compact, high-gain, microwave and millimeter-wave amplifiers. ${ }^{1,2}$ A new class of microelectronic and vacuum tube microwave and millimeterwave amplifiers, called deflectrons, ${ }^{3,4}$ has been proposed, which achieves modulation through the deflection of the beam. The deflection concept, together with others recently proposed, ${ }^{5,6}$ utilize the deflection of a low-voltage electron beam generated from gridded field-emitter arrays to amplify microwave signals or to provide a bunched electron beam source.

In the deflectron amplifier, the electron beam is emitted continuously from the field-emitter arrays and beam modulation is provided by the transverse electric field of a set of deflectors. This is in contrast to the prevalent method of longitudinal pulse generation by grid voltage modulation of gridded field-emitter arrays. The deflection amplifier concept bypasses the restriction on transconductance imposed by grid-modulated field-emitter arrays. Additional advantages include the following: (i) the capability of using field-emitter arrays with high resistive tips without lowering the frequency response, thereby yielding (ii) more uniform emission and tip blow-up protection, (iii) smaller capacitance in the field-emitter array circuit, and (iv) frequency doubling and harmonics operation.

Deflection tubes with thermionic cathodes have been studied for a long time. ${ }^{7,8}$ Deflection oscillators at $750 \mathrm{MHz}$ were successfully implemented 30 years ago. ${ }^{9}$ With the recent advances in gridded cold field-emitter arrays, amplifiers based on the deflection of electron beams need to be reexamined.

A compact deflectron used to amplify microwaves requires an electron beam with small emittance, small energy spread, high-current density, and source packageability in a small structure. Recently, field-emitter array current densities of up to $1600 \mathrm{~A} / \mathrm{cm}^{2}$ have been achieved, ${ }^{10}$ four orders of magnitude larger than quoted in Ref. 7. Wedge and edge emitters are also applicable for a deflectron. A measure of the focusability of the beam in the $x$ direction (beam deflection direction) is the emittance. In the paraxial limit, the phasespace area occupied by $\left(x, v_{x}\right)$ is the $x$ directed normalized emittance $\pi \epsilon_{n, x}$. The normalized rms emittance associated with a single cone type field-emitter tip with lens is found to be as small as a few $10^{-4} \pi \mathrm{mm}$ mrad calculated by computer code EGUN2. ${ }^{11}$ This very small emittance allows the construction of an array of field emitters with collimating lenses capable of focusing the electron beam to a very narrow line, necessary for the deflection concept. The energy spread from field-emitter arrays is on the order of $\Delta E \lesssim 0.2$ $\mathrm{eV}$, while the energy spread from thermionic cathodes is typically on the order of $1 \mathrm{eV}$.

A schematic of a deflection amplifier is shown in Fig. 1. The incoming electron beamlet is at a low-energy, typically on the order of tens of $\mathrm{eV}$. There are many possible anode configurations. ${ }^{3}$ The required modulation voltage is on the order of a tenth of volts to a few volts. This small drive voltage enables high-frequency operation with high gain.

If we remove anode 2 in Fig. 1, then the combination of the deflectors A, B, and anode 1 may be regarded as an electron source, which generates bunched electrons at high frequencies. The modulated beams from these sources replace the continuously emitting cathodes and the bunching

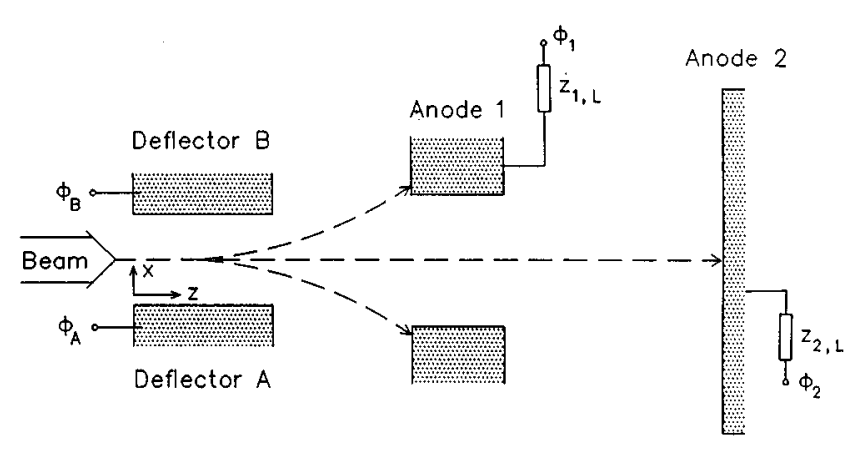

FIG. 1. Schematic of a deflection amplifier. 
cavities of conventional power amplifiers, leading to the following advantages: (i) high efficiency, (ii) wide bandwidth, (iii) light weight and compactness, as discussed above.

In this letter, we establish the frequency, $f_{T}$, at which the short-circuit current gain attains unit magnitude. The result is valid for arbitrary deflector lengths, distances between the deflector and the anode slit, and applied voltages at the anode and deflectors, and is indicative of the frequency of operation. We assume there is no overlap of the deflector with the emitter structure, and that the signal is supplied by a stripline source. Practical issues, such as excitations of parasitics, await further studies.

We first establish that the maximum current that can be propagated through the deflector per unit length in the $y$ direction:

$$
\frac{\Delta I_{\max }}{\Delta y} \simeq \frac{8 \epsilon_{0}}{3 D}\left(\frac{2 e}{3 m_{e}}\right)^{1 / 2}\left(V_{i}\right)^{3 / 2},
$$

where $e$ is the absolute value of the elementary charge, $m_{e}$ is the electron mass, $\epsilon_{0}$ is the free-space permeability, $e V_{i}$ is the energy of electrons in units of $\mathrm{eV}$, and $D$ is the gap between the deflectors and the beam is located midway between the deflectors.

We next utilized EGUN2 to verify the calculation of limiting current. For example, with an electron beam energy of $e V_{i}=10 \mathrm{eV}$, a deflector separation of $D=8 \mu \mathrm{m}$ and the potentials of the deflectors at $10 \mathrm{~V}$, the limiting current based on Eq. (1) is $\Delta I_{\max } / \Delta y=30 \mu \mathrm{A} / \mu \mathrm{m}$. EGUN2 simulations show that space-charge effects begin to be important at $\Delta I / \Delta y \simeq 10$ $\mu \mathrm{A} / \mu \mathrm{m}$ and become detrimental at $\Delta I / \Delta y \simeq 20 \mu \mathrm{A} / \mu \mathrm{m}$, consistent with the theory.

To determine the appropriate length of the deflector, we need to know the admittance and the transverse velocity of the beam as a function of the length of the deflector. We assume that the electric field in the deflector region has the form $\mathbf{E}=E_{0} \sin (\omega t) \hat{\mathbf{e}}_{x}$, where $\omega=2 \pi f$ and $f$ is the frequency. We assume that there is no fringe field at the end of the deflectors and use the initial conditions $x(z=0, t)=0$ and $v_{x}(z=0, t)=0$ for the beam entering the deflectors.

The instantaneous shape of the beam in the deflector is

$$
\begin{aligned}
x(z, t)= & \left(e / \omega^{2} m_{e}\right) E_{0}\left[\sin (\omega t)-\sin \omega\left(t-z / v_{z}\right)\right. \\
& \left.-\left(\omega z / v_{z}\right) \cos \omega\left(t-z / v_{z}\right)\right],
\end{aligned}
$$

where $v_{z}$ is the axial velocity. The average electron displacement of all the charges between the deflectors is

$$
\begin{aligned}
X(t) & =\frac{1}{L} \int_{0}^{L} x(z, t) d z \\
& =\frac{e}{\omega^{2} m_{e}} \frac{E_{0}}{\theta}[G(\theta) \cos (\omega t)-B(\theta) \sin (\omega t)],
\end{aligned}
$$

where

$$
\begin{aligned}
& G(\theta)=2(1-\cos \theta)-\theta \sin \theta, \\
& B(\theta)=-\theta(1+\cos \theta)+2 \sin \theta,
\end{aligned}
$$

$\theta=\omega L / v_{z}$ and $L$ is the length of the deflector (Fig. 2).

The admittance is defined as

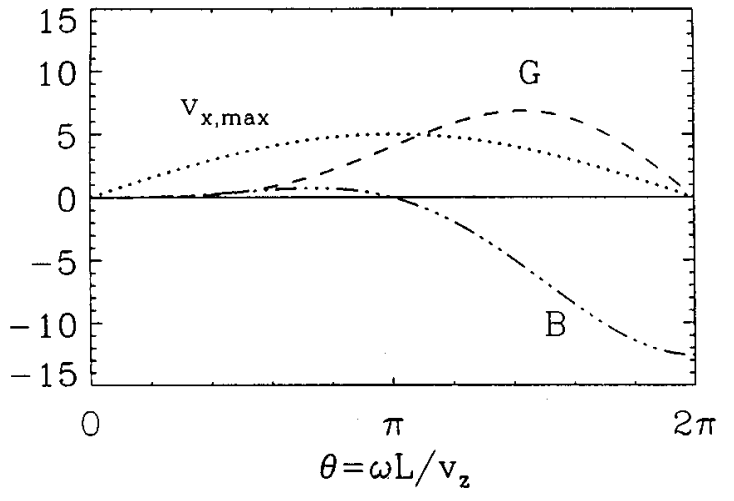

FIG. 2. Plot of $G, B$, and the maximum $x$ component of velocity $v_{x, \max }$ (in arbitrary units) at the end of the deflector as a function of the normalized deflector length $\theta$.

$$
Y=\hat{I} \hat{V}^{*} /|\hat{V}|^{2}
$$

where $I=\hat{I} \exp (i \omega t)+$ c.c. and similarly for $V$. The conductance is $\operatorname{Re}(Y) \propto G$ and the susceptance is $\operatorname{Im}(Y) \propto B$.

The maximum $x$ component of velocity at the end of the deflector is

$$
v_{x, \max }=\frac{2 e}{\omega m_{e}} E_{0} \sin \left(\frac{\theta}{2}\right) .
$$

Figure 2 plots the $B, G$, and the $x$ component of velocity $v_{x, \max }$ (in arbitrary units) at the end of the deflector as a function of $\theta$. We restrict the length of the deflector to be $0<\theta \leqslant \pi$.

An anode with a slit can produce a chopped beam, when a transverse rf electric field is imposed across the deflector. We assume that the beam has a thickness of $\delta x$ in the deflector and a thickness of $\Delta x$ at the anode slit. The extracted beam has the maximum modulation when the beam moves by $\Delta x$ across the edge of the anode slit. The anode that chops the beam is assumed to be located at a distance $\bar{n} L$ from the end of the deflector. It is held at a potential $\Delta \Phi$ larger than the deflector potential to reduce the space-charge effect. We obtain the expression for the minimum amplitude of the oscillating potential $\Delta V=E_{0} D$ between the deflectors required to obtain full beam modulation.

The time required for the beam to propagate from the end of the deflector to the anode is

$$
t=n L / v_{z},
$$

where $n=\bar{n}[2(\sqrt{1+\zeta}-1) / \zeta]$ can be thought of as an adjustment to axial length when $\Delta \Phi \neq 0$ and $\zeta=\Delta \Phi / V_{i}$ and we have used $v_{z}=\left(2 e / m_{e}\right)^{1 / 2}\left(V_{i}\right)^{1 / 2}$.

The anode can be constructed so that the current at the anode changes by $\Delta I / \Delta y$, when the beam at the anode is reflected by $\Delta x$. Combining Eqs. (7) and (8), we obtain the voltage difference between the deflectors that is required to deflect the beam by the beam thickness $\Delta x$ at the anode:

$$
\Delta V=\frac{2 \pi^{2}}{(\theta / 2) \sin (\theta / 2)} \frac{m_{e}}{e} D f^{2} L \theta_{\epsilon},
$$


where $\theta_{\epsilon}=(\Delta x / 2) / n L$ is the divergence angle of the beam. Beam collimation and focusing are determined by normalized emittance, a conserved quantity. The normalized emittance of a focused beam can be defined as

$$
\epsilon_{n, x}=(2 / \pi) \delta x \beta \theta_{\epsilon},
$$

where $\beta \simeq v_{z} / c$ and $c$ is the speed of light.

To calculate power gain, we postulate that the frequency at which the short-circuit current gain attains unit magnitude, $f_{T}=g_{m} / 2 \pi C$, may still be used as a figure of merit in the short-circuit current-gain-bandwidth product for deflectrons, where $g_{m}=\Delta I / \Delta V$ is the transconductance, and $C$ is the input capacitance.

The capacitance depends on space-charge loading. If stray capacitance is neglected, then

$$
C=C_{0}(1+\xi)
$$

where

$$
\begin{aligned}
& C_{0}=\epsilon_{0} w L / D, \\
& \xi=\frac{\sqrt{e / m_{e}}}{4 \sqrt{2} \pi^{2} \epsilon_{0}} \frac{1}{f^{2} D} \frac{\sqrt{B^{2}(\theta)+G^{2}(\theta)}}{\theta} \frac{\Delta I}{\Delta y}\left(V_{i}\right)^{-1 / 2},
\end{aligned}
$$

and $w$ is the width of the beam in the deflector. The value of $\xi$ approaches 0 as $\theta$ approaches 0 , since $\left(\sqrt{B^{2}(\theta)+G^{2}(\theta)} / \theta\right) \simeq \theta^{2} / 6$ for $\theta \ll 1$.

Substituting Eq. (9) for $\Delta V$ and Eq. (11) for $C$ into the definition of $f_{T}$, we obtain

$$
f_{T}=\frac{1}{8 \pi} \frac{1}{\epsilon_{0}} \frac{\sin (\theta / 2)}{\theta / 2} \frac{1}{1+\xi}\left(\frac{1}{\theta_{\epsilon} V_{i}} \frac{\Delta I}{\Delta y}\right) .
$$

We note that $f_{T}$ is a function of the electron beam parameters, i.e., electron beam energy, line current density, and beam divergence angle, independent of total current and the total beamwidth $w$. As $\theta$ approaches $0[\sin (\theta / 2) /(\theta / 2)] /(1+\xi)$ approaches 1 and $f_{T}$ approaches a maximum.

We evaluate Eq. (14) for the following numerical examples. We consider a line current density of $\Delta I / \Delta y=20$ $\mu \mathrm{A} / \mu \mathrm{m}, \bar{n}=3, \delta x=5 \mu \mathrm{m}$, and $D=10 \mu \mathrm{m}$. EGUN2's simulation shows that the effect of space charge on beam propagation becomes negligible when $J=\left(\Delta I_{\max } / \Delta I\right) \geqslant 4$. Taking $J=4$, i.e., $\Delta I_{\max } / \Delta y=80 \mu \mathrm{A} / \mu \mathrm{m}$, we obtain $e V_{i}=21.4 \mathrm{eV}$ and $\beta=9.15 \times 10^{-3}$. For $\Delta \Phi=62.9 \mathrm{~V}$, we obtain $n=2.01$. Assuming a beam divergence angle of $2^{\circ}, \theta_{\epsilon}=3.49 \times 10^{-2}$. (i) For $\theta$ approaching $0, f_{T}$ approaches $120 \mathrm{GHz}$. (ii) For $\theta=\pi / 4$, the deflector length is $L=17.0 \mu \mathrm{m}$ for $f=20 \mathrm{GHz}$. The required deflection voltage is $\Delta V=1.76 \mathrm{~V}$. The capacitance loading coefficient is $\xi=9.28 \times 10^{-2}$. The frequency $f_{T}$ is $107 \mathrm{GHz}$. (iii) For $\theta=\pi / 8$ and an electron beam with the same energy, normalized emittance, and current, the deflector length is $L=17.0 \mu \mathrm{m}$ for $f=10 \mathrm{GHz}$. The required deflection voltage is $\Delta V=1.74 \mathrm{~V}$. The capacitance loading coefficient is $\xi=2.35 \times 10^{-2}$. The frequency $f_{T}$ is $117 \mathrm{GHz}$.

We consider a smaller deflector gap, $D=6 \mu \mathrm{m}$ but the same line current density of $\Delta I / \Delta y=20 \mu \mathrm{A} / \mu \mathrm{m}$. For $\Delta I_{\text {max }} / \Delta y=80 \mu \mathrm{A} / \mu \mathrm{m}$, we obtain $e V_{i}=15.2 \mathrm{eV}$ and $\beta=7.72 \times 10^{-3}$. Again, assuming a beam divergence angle of $2^{\circ}, \theta_{\epsilon}=3.49 \times 10^{-2}$. For $\theta$ approaching $0, f_{T}$ approaches 169 $\mathrm{GHz}$.

As long as the emittance $\epsilon_{n, x}$ remain small, the reduction in current density can be partially offset by a decrease in electron beam energy $e V_{i}$. For example, for $\Delta I / \Delta y=5 \mu \mathrm{A}$, $J=4$, and $D=6 \mu \mathrm{m}$, then $V_{i}=9.6 \mathrm{~V}$. For short deflector length, i.e., $\theta$ approaching $0, f_{T}$ approaches $67 \mathrm{GHz}$.

Since $f_{T}$ depends only on electron beam energy, current density, and normalized emittance, and is independent of total current and total beamwidth, the power output can be adjusted by connecting multiple units of deflectors together in an appropriate manner so as to obtain the necessary total current.

Various designs of beam propagation and collimation with cone emitters and edge emitters have been obtained by EGUN with normalized emittance on the order of few $10^{-4} \pi$ $\mathrm{mm} \mathrm{mrad}$ to $10^{-3} \pi \mathrm{mm}$ mrad. Time-dependent particle simulations $^{3}$ were performed by the computer code MAGIC ${ }^{12}$ to assess the effect of space charge and beam loading on the anode. The emission properties of field-emitter arrays are superior to those of thermionic cathodes in current density. If the fabrication of the emitters along with the beam collimation lenses is achievable, the results indicate that fieldemitter arrays have the potential of making wideband deflection amplifiers at frequencies up to tens of $\mathrm{GHz}$.

Work is supported by the Defense Sciences Office of ARPA. We would like to express special thanks to Dr. Robert Adler of Zenith and Dr. Sidney T. Smith of NRL.

${ }^{1}$ R. K. Parker and R. H. Abrams, 1992 Government Microcircuit Applications Conference (GOMAC), Digest of Papers (U.S. GPO, Washington, 1992), Vol. XVIII, pp. 29-32.

${ }^{2}$ C. A. Spindt, C. E. Holland, A. Rosengreen, and I. Brodie, J. Vac. Sci. Technol. B 11, 468 (1993).

${ }^{3}$ C. M. Tang, Y. Y. Lau, and T. A. Swyden, J. Vac. Sci. Technol. B (to be published)

${ }^{4}$ C. M. Tang, Y. Y. Lau, and T. A. Swyden, Technical Digest of the 1993 IEEE International Electronics Devices Meeting, Washington, DC, 5-8 December 1993 (unpublished), pp. 757-760.

${ }^{5}$ H. F. Gray, U.S. Patent 5,030,895 (9, July, 1991).

${ }^{6}$ W. L. Ohlinger and L. R. Barnett, , Technical Digest of the Fourth International Vacuum Microelectronics Conference, Nagahama, Japan, 22-24 August 1991 (unpublished), pp. 110-111.

${ }^{7}$ G. R. Kilgore, RCA Rev. VIII, 480 (1947).

${ }^{8}$ R. Adler, U.S. Patent No. 2,553,735 (22 May, 1951).

${ }^{9}$ R. Adler (private communications).

${ }^{10}$ C. Bozler, C. T. Harris, S. Rabe, D. D. Rathman, M. A. Hollis, and H. I. Smith, J. Vac. Sci. Technol. B 12, 629 (1994).

${ }^{11}$ W. B. Herrmannsfeldt, EGUN-An Electron Optics and Gun Design Program, SLAC Report No. 331, 1988.

${ }^{12}$ MAGIC Code, Mission Research Corporation, Newington, VA 22122. 